\title{
EFEITO DOS MÉTODOS DE COCÇÃO SOBRE A COMPOSIÇÃO QUÍMICA E PERFIL LIPÍDICO DE FILÉS DE TILÁPIA DO NILO (Oreochromis niloticus Linnaeus 1757)
}

\author{
Effects of cooking method on chemical composition and fat profile of Nile Tilapia \\ (Oreochromis niloticus Linnaeus, 1757) fillets
}

\author{
Milena Wolff Ferreira ${ }^{1}$, Maria Cristina Bressan ${ }^{2}$, Xisto Rodrigues de Souza ${ }^{3}$, Josye Oliveira e Vieira ${ }^{4}$, \\ Peter Bitencourt Faria ${ }^{5}$, Patrícia Lopes Andrade ${ }^{6}$
}

\begin{abstract}
RESUMO
No presente trabalho foi avaliado o efeito de métodos de cocção na composição química, teor de colesterol e totais de ácidos graxos de filés de Tilápias do Nilo. Os tratamentos foram cozimento em água (CA), fritura em óleo de soja (FO), assado em forno elétrico (FE), assado em forno microondas (MO), e um grupo testemunha (filés crus). O delineamento foi inteiramente casualizado, cada tratamento com 7 peixes e a unidade experimental foi um peixe ( 2 filés). Houve aumento $(\mathrm{P}<0,05)$ na matéria seca, quando os filés preparados (CA, FE, MO, e FO com médias de 23,27 \%; 25,44 \%; 31,93\% e 33,44\%, respectivamente) foram comparados com os crus $(20,61 \%)$. Os filés FO apresentaram lipídeos totais mais elevados $(\mathrm{P}<0,05)(17,36 \%)$, que os demais tratamentos CA, FE, MO e Crus $(6,94 \% ; 9,80 \% ; 8,66 \%$ e $5,92 \%$, respectivamente). Os métodos de cocção aumentaram $(\mathrm{P}<0,05)$ o colesterol dos filés $(46,89$; 46,46; 54,78 e 57,28 mg 100 $\mathrm{g}^{-1}$ para CA, FE, FO e MO, respectivamente) com relação aos crus $(33,00 \mathrm{mg} / 100 \mathrm{~g})$. Os filés FO (P<0,05) apresentaram menores totais de ácidos graxos saturados e monoinsaturados e mostraram maior percentual de ácidos graxos poliinsaturados, quando comparados aos demais grupos. O cozimento aumenta a matéria seca, resultado da perda de água dos filés e aumento na concentração dos demais constituintes. O tratamento frito em óleo causa uma incorporação do meio (óleo de fritura) aos filés e aumenta os lipídeos totais, alterando o perfil de ácidos graxos dos filés de peixes fritos.
\end{abstract}

Termos para indexação: Peixe, ácidos graxos, colesterol.

\section{ABSTRACT}

In this work, the effects of different cooking methods of Nile tilapia fillets on their chemical composition, cholesterol content and total fatty acids, were studied. Cooking methods evaluated were water boiling (WB), frying in soybean oil (SO), broiling in an electric oven (EO), cooking in a microwave oven (MO) and a control group (raw fillets). The experiment was conducted as a completely randomized design, with the fish (two fillets/fish) considered as the experimental unit, and seven experimental units were used per treatment. Dry matter increased $(\mathrm{P}<0.05)$ in cooked fillets $(23.27 \% \mathrm{WB}, 25.44 \% \mathrm{EO}, 31.93 \% \mathrm{MO}$ and $33.44 \% \mathrm{SO})$ when compared with raw fillets $(20.61 \%)$. Total lipids were higher $(\mathrm{P}<0.05)$ in SO fillets $(17.36 \%)$, than in all the other treatments $(6.94$ $\% \mathrm{WB}, 9.80 \% \mathrm{EO}, 8.66 \% \mathrm{MO}$ and $5.92 \%$ raw fillets). All cooking methods increased $(\mathrm{P}<0.05)$ cholesterol content of the fillets

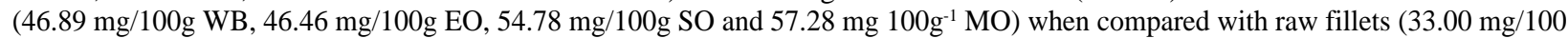
g). Relative to all the other treatments, FO fillets had $(\mathrm{P}<0.05)$ lower values for saturated and monounsaturated fatty acids, and a higher percentage of polyunsaturated fatty acids. Overall, water boiling results in an increase in dry matter due to water loss in the cooking process, with higher concentration of the remaining constituents. Cooking by oil frying leads to the incorporation of oil in the fillets, with an increase in total lipids and a change of the fatty acid profile in fried fish.

Index terms: Fish, fatty acids, cholesterol.

(Recebido em 23 de março de 2005 e aprovado em 20 de outubro de 2005)

\section{INTRODUÇÃO}

O crescimento da aqüicultura brasileira é resultado da maior demanda do produto decorrente da maior aceitação do produto no mercado e da imagem favorável propagada pelos profissionais da área da saúde. Nesse sentido, algumas pesquisas relacionam hábitos alimentares, níveis de colesterol e de triacilgliceróis no sangue a doenças cardiovasculares (HUTTER \& LANG, 1996;

\footnotetext{
'Mestre em Ciência dos Alimentos, aluna de doutorado no Departamento de Zootecnia/DZO - Universidade Federal de Lavras/UFLA - Cx. P. 3037 37200-000 - Lavras, MG - milenawolff@bol.com.br

2Professora, Dra. do Departamento de Ciência dos Alimentos/DCA - Universidade Federal de Lavras/UFLA - Cx. P. 3037 - $37200-000$ - Lavras, MG bressan@ufla.br

${ }^{3}$ Doutor em Ciência dos Alimentos - Professor do Centro Federal de Educação Tecnológica de Cuiabá/CEFET - BR 364 , Km 329 - São Vicente da Terra - 78106-000 - Santo Antônio do Leverger, MT - xistorod@yahoo.com.br

${ }^{4}$ Mestre em Ciências dos Alimentos pelo Departamento de Ciência dos Alimentos/DCA -Universidade Federal de Lavras/UFLA - Cx. P. 3037 37200-000 - Lavras, MG - josyevet@yahoo.com.br

${ }^{5}$ Mestre em Ciência dos Alimentos, Professor do Centro Federal de Educação Tecnológica de Cuiabá/CEFET - BR 364, Km 329 - São Vicente da Terra 78106-000 - Santo Antônio do Leverger, MT - peterbfvet@yahoo.com.br

${ }^{6}$ Aluna de mestrado do Departamento de Ciência dos Alimentos/DCA - Universidade Federal de Lavras/UFLA - Cx. P. 3037 - $37200-000$ - Lavras, MG patricialandrade@zipmail.com.br
} 
SINCLAIR, 1953). Com isso uma grande atenção é dada aos níveis de ácidos graxos poliinsaturados e a relação ômega3/ômega-6 presentes na dieta (SIMOPOULOS, 1991).

As embalagens dos alimentos trazem rótulos com apresentação da informação nutricional, de acordo com o estabelecido pela Agência Nacional de Vigilância Sanitária, por meio da Resolução RDC n 40, de 21 de março de 2001, isso permite o acesso dos consumidores às informações sobre a composição química dos alimentos, possibilitando escolhas mais saudáveis em relação à dieta. Entretanto, as informações referentes ao conteúdo nutricional dos alimentos após o preparo não estão disponíveis aos consumidores, e são pouco conhecidas.

Os processos de cozimento podem alterar as características dos produtos in natura, pois: inicialmente ocorre a perda de água, que promove a concentração dos nutrientes; seguido da incorporação de substâncias provenientes do meio de cocção (ex. óleo, água, temperos) e também perdas para esse meio. O calor, por si só, produz diversas modificações nos componentes químicos do produto in natura, incluindo: composição de ácidos graxos, teores de vitaminas, conteúdo de colesterol, teores e forma das proteínas (BADIANI et al., 2002; POTTER \& HOTCHKISS, 1995; ROSA, 2003). Entretanto, trabalhos que relatam o efeito do cozimento sobre a estabilidade dos ácidos graxos são escassos.

Os objetivos deste trabalho foram avaliar o efeito dos métodos de cocção em filés de Tilápia do Nilo (Oreochromis niloticus) na composição química, teor de colesterol e totais de ácidos graxos.

\section{MATERIAL E MÉTODOS}

Foram utilizados 35 tilápias do Nilo (Oreochromis niloticus), pesando em média 700 gramas, criadas em tanque de terra e alimentadas com ração comercial contendo em média 19,98\% de ácidos graxos saturados, $36,25 \%$ de ácidos graxos monoinsaturados e 0,84\% de ácidos graxos poliinsaturados. Os fito e zooplânctons encontrados no tanque de criação apresentaram $24,8 \%$ de ácidos graxos saturados, 24,78\% de ácidos graxos monoinsaturados e $31,37 \%$ de ácidos graxos poliinsaturados.

Os peixes, na ocasião do abate, foram submetidos à depuração por 24 horas, atordoados por choque térmico, seguido do rompimento do cordão neural. Os filés, retirados logo após o abate, foram lavados com água clorada, resfriados e mantidos a $-20^{\circ} \mathrm{C}$ por dois dias, até a aplicação dos tratamentos.
Os exemplares foram divididos aleatoriamente em 5 grupos, 4 métodos de cocção e um grupo testemunha (cru), que foram:

a) cozido em água (CA), cada filé foi colocado em uma panela com $500 \mathrm{~mL}$ de água e cozidos;

b) frito em óleo (FO), os filés foram fritos em óleo quente, virados a cada minuto, foram utilizados $200 \mathrm{~mL}$ de óleo de soja por filé;

c) assado em forno elétrico $(\mathrm{FE})$, os filés foram assados em forno elétrico a $150{ }^{\circ} \mathrm{C}$, por aproximadamente 20 minutos;

d) assado em forno microondas (MO), os filés foram assados em forno de microondas por 6 minutos, virados a cada 3 minutos.

A temperatura interna dos filés foi monitorada em todos os processos e o cozimento foi estendido até a temperatura interna dos filés alcançarem $72^{\circ} \mathrm{C}$. Os 2 filés de cada exemplar, após os tratamentos, foram homogeneizados para compor a amostra.

A composição química (umidade, proteína, lipídeos totais e cinzas) foi analisada seguindo metodologia da AOAC (1990).

O perfil lipídico do óleo de soja utilizado é apresentado na Tabela 1 .

TABELA 1 - Perfil de ácidos graxos do óleo de soja utilizado nos filés fritos.

\begin{tabular}{llc}
\hline Ácidos Graxos & Óleo de soja (\%) \\
\hline AGS & Mirístico & 0,07 \\
& Palmítio & 10,90 \\
Esteárico & 3,07 \\
Total & 14,04 \\
\hline AGM & Palmitoleico & 0,10 \\
Oléico & 19,88 \\
Eicosanóico & 0,18 \\
Total & 20,16 \\
\hline AGP & Linoléico & 57,71 \\
$\gamma$ linolênico & 6,33 \\
a linolênico & - \\
Araquidônico & - \\
Eicosapentaeníco & 0,33 \\
Docosahexaenóico & - \\
Total & 67,37 \\
\hline
\end{tabular}


Para a realização das análises de colesterol e ácidos graxos, os lipídeos foram extraídos seguindo a metodologia de Folch et al. (1957), adaptada para amostras de 5 gramas, que foi homogeneizada em $50 \mathrm{~mL}$ de clorofórmio/metanol (2:1). A amostra homogeneizada foi filtrada para funil de separação de $250 \mathrm{~mL}$, permanecendo em repouso por 2 horas para a separação física. A fração apolar do homogeneizado, contendo lipídeos e clorofórmio, foi recolhida e a fração polar descartada. A fração apolar foi submetida à nova separação por 12 horas; dessa segunda separação, a fração apolar foi recolhida em balão volumétrico e foi adicionado clorofórmio até completar $50 \mathrm{~mL}$. Desse extrato, $5 \mathrm{~mL}$ foram retirados para a determinação do colesterol e $5 \mathrm{~mL}$ para a análise do perfil de ácidos graxos.

A determinação do colesterol foi realizada por colorimetria, segundo Bragagnolo \& Rodriguez-Amaya (1995). O perfil de ácidos graxos foi obtido por cromatografia gasosa, segundo Firestone (1998). As amostras foram submetidas à cromatografia gasosa e injetadas manualmente em cromatógrafo a gás (marca Shimadzu, modelo GC-17A), equipado com detector de ionização de chama, injetor split na razão de 1:00, coluna capilar de polietileno-glicol DB-Wax (30 m;0,25 mm; $0,25 \mu \mathrm{m})$ e acoplado a um software desenvolvido pela Shimadzu. As condições cromatográficas foram: temperatura inicial da coluna $190{ }^{\circ} \mathrm{C}$ por 15 minutos, aumentada a uma taxa de $5^{\circ} \mathrm{C} /$ minuto até a temperatura final da coluna de $220^{\circ} \mathrm{C}$, permanecendo nessa temperatura por 22 minutos; temperatura do injetor $250{ }^{\circ} \mathrm{C}$; e, temperatura do detector $260^{\circ} \mathrm{C}$. O gás de arraste utilizado foi o nitrogênio em fluxo de $0,7 \mathrm{~mL} /$ minuto.

O delineamento experimental foi inteiramente casualizado com 7 repetições (formada cada uma por 2 filés), e os métodos de cocção (cozido em água, frito em óleo de soja, assado em forno elétrico e microondas) considerados como tratamentos. O modelo estatístico é apresentado a seguir:

$$
Y_{i j}=\mu+t_{i}+e_{i j}
$$

$Y_{i j}=$ observação no tratamento $i$, na repetição $j$;

$i=$ média geral do experimento;

$t_{i}=$ efeito do tratamento $i, \operatorname{com} i=1,2,3,4,5$;

$e_{i j}=$ erro experimental associado à observação $Y_{i j}$, que

por hipótese tem distribuição normal com média igual a zero e variância $\sigma^{2}$.

Os dados foram submetidos à análise de variância pelo programa estatístico SISVAR (FERREIRA, 2000), e as médias dos tratamentos comparadas pelo teste de Tukey, a $5 \%$ de significância.

\section{RESULTADOS E DISCUSSÃO}

Os métodos de cocção influenciaram $(\mathrm{P}<0,05)$ os percentuais de matéria seca, proteína, lipídeos totais e cinzas dos filés de tilápia (TABELA 2). Isso mostra que os métodos de cozimento determinam alterações na composição química, quando comparados aos filés cozidos ou assados com os filés crus. Esses achados confirmam as informações de Badiani et al. (2002), Potter \& Hotchkiss (1995) e Rosa (2003).

A matéria seca no tratamento dos filés CA $(20,61$ $\%)$ foi inferior as médias encontradas nos filés dos tratamentos CA e FE (23,27 e 25,44 \%, respectivamente), que por sua vez foram inferiores as médias encontradas nos filés MO e FO (31,93 e 33,44 \%, respectivamente). Isso demonstra que os filés assados no forno de microondas e fritos em óleo apresentam percentuais de matéria seca mais elevados, que os demais tratamentos ou menor taxa de umidade, mostrando que os métodos MO e FO determinam perdas de água mais elevadas, durante o cozimento, do que os métodos CA e FE. Resultados semelhantes foram reportados por Gokoglu et al. (2004) em filés de truta que observaram taxas de umidade mais baixas em filés fritos em óleo e assados em microondas (62,69 e 63,52\%, respectivamente), do que os filés assadps e, forno convencional, grelhados e cozidos em água $(65,30 ; 65,83$ e $69,16 \%$, respectivamente). Entretanto Gall et al. (1983), trabalhando com filés de garoupa, caranha vermelha, pampo de Flórida e cavalinha, observaram menor umidade nos filés fritos do que os filés assados em forno convencional e microondas e grelhados.

Os métodos de cocção influenciaram $(\mathrm{P}<0,05)$ os percentuais de proteína dos filés. Valores mais elevados de proteínas, foram observados nos filés cozidos em água e assados em forno elétrico. Os filés crus, assados em forno microondas e fritos em óleo apresentaram teores de proteína semelhantes. Puwastien et al. (1999), estudando dois métodos de cocção (cozido em água e frito por imersão) em três espécies de peixes (tilápia, bagre africano e catfish), encontraram médias mais elevadas nos peixes submetidos à fritura e valores mais baixos nos peixes cozidos em água. Os autores explicam esse comportamento em função da incorporação do óleo da fritura que resultou, trabalho desses autores, num percentual mais elevados de lipídeos totais.

Ciênc. agrotec., Lavras, v. 31, n. 3, p. 798-803, maio/jun., 2007 
TABELA 2 - Valores médios da composição química (\%), de colesterol (mg/100 g) e dos totais de ácidos graxos (\%), seguidos dos respectivos desvios padrão.

\begin{tabular}{lrrrrr}
\hline & \multicolumn{5}{c}{ Tratamentos } \\
\cline { 2 - 6 } & \multicolumn{1}{c}{ CRU } & CA & FE & MO & FO \\
\hline Matéria Seca & $20,61 \pm 0,62^{\mathrm{c}}$ & $23,27 \pm 0,46^{\mathrm{b}}$ & $25,44 \pm 0,88^{\mathrm{b}}$ & $31,93 \pm 1,06^{\mathrm{a}}$ & $33,44 \pm 1,84^{\mathrm{a}}$ \\
Proteína & $79,16 \pm 2,45^{\mathrm{b}}$ & $89,79 \pm 3,18^{\mathrm{a}}$ & $85,05 \pm 1,96^{\mathrm{a}}$ & $83,33 \pm 2,39^{\mathrm{b}}$ & $74,45 \pm 1,98^{\mathrm{c}}$ \\
Lipídeos totais & $5,92 \pm 0,59^{\mathrm{c}}$ & $6,94 \pm 0,63^{\mathrm{c}}$ & $9,80 \pm 0,88^{\mathrm{b}}$ & $8,66 \pm 0,56^{\mathrm{b}}$ & $17,36 \pm 1,52^{\mathrm{a}}$ \\
Cinzas & $5,01 \pm 0,08^{\mathrm{a}}$ & $3,94 \pm 0,8^{\mathrm{c}}$ & $4,60 \pm 0,12^{\mathrm{b}}$ & $5,18 \pm 0,05^{\mathrm{a}}$ & $4,28 \pm 0,20^{\mathrm{b}}$ \\
Colesterol & $33,00 \pm 1,82^{\mathrm{c}}$ & $46,89 \pm 1,17^{\mathrm{b}}$ & $46,46 \pm 1,66^{\mathrm{b}}$ & $54,78 \pm 1,31^{\mathrm{a}}$ & $57,28 \pm 3,12^{\mathrm{a}}$ \\
Ácidos Graxos & & & & & \\
AGS & $32,55 \pm 0,88^{\mathrm{a}}$ & $31,92 \pm 1,25^{\mathrm{a}}$ & $31,51 \pm 1,03^{\mathrm{a}}$ & $31,45 \pm 2,47^{\mathrm{a}}$ & $16,30 \pm 0,71^{\mathrm{b}}$ \\
AGM & $40,32 \pm 2,07^{\mathrm{a}}$ & $38,35 \pm 2,98^{\mathrm{a}}$ & $40,87 \pm 1,80^{\mathrm{a}}$ & $40,57 \pm 7,62^{\mathrm{a}}$ & $25,52 \pm 1,21^{\mathrm{b}}$ \\
AGP & $22,97 \pm 2,66^{\mathrm{b}}$ & $23,49 \pm 2,32^{\mathrm{b}}$ & $22,30 \pm 2,32^{\mathrm{b}}$ & $23,53 \pm 2,47^{\mathrm{b}}$ & $57,00 \pm 2,02^{\mathrm{a}}$ \\
AGP/AGS & 0,71 & 0,74 & 0,70 & 0,75 & 3,50 \\
Total $\omega 3$ & $3,03 \pm 0,45^{\mathrm{b}}$ & $3,29 \pm 0,52^{\mathrm{b}}$ & $2,86 \pm 0,43^{\mathrm{b}}$ & $3,04 \pm 1,20^{\mathrm{b}}$ & $6,06 \pm 0,24^{\mathrm{a}}$ \\
Total $\omega 6$ & $19,93 \pm 2,42^{\mathrm{b}}$ & $20,19 \pm 2,47^{\mathrm{b}}$ & $19,44 \pm 2,00^{\mathrm{b}}$ & $20,49 \pm 3,84^{\mathrm{b}}$ & $50,94 \pm 1,88^{\mathrm{a}}$ \\
$\omega 3 / \omega 6$ & 6,64 & 6,31 & 6,94 & 6,88 & 8,42 \\
\hline
\end{tabular}

Médias seguidas de letras diferentes, na linha, diferem estatisticamente entre si, pelo Teste de Tukey, a $5 \%$ de significância.

$\mathrm{CA}=$ cozido em água; $\mathrm{FO}=$ frito em óleo; $\mathrm{FE}=$ assado em forno elétrico; $\mathrm{MO}=$ assado em forno microondas

AGS = ácidos graxos saturados; AGM = ácidos graxos monoinsaturados; $\mathrm{AGP}=$ ácidos graxos poliinsaturados.

Os filés do tratamento FO mostraram $(\mathrm{P}<0,05)$ médias de lipídeos totais $(17,36 \%)$ mais elevados que os filés do tratamento $\mathrm{FE} \mathrm{e} \mathrm{MO}(9,80$ e $8,66 \%$, respectivamente), que por sua vez foram mais elevadas que os filés do tratamento CA e Cru (6,94 e 5,92 \%, respectivamente). Esses resultados mostram que os aumentos nos percentuais de lipídeos dos filés cozidos, quando comparados aos filés crus foi de 17,22; 65,54; 46,28 e 193,24 \% para filés cozidos em água, assados em forno elétrico, assado em forno de microondas e frito em óleo, respectivamente. Ou seja, os métodos de cocção determinaram aumento nos lipídeos totais (CA, FE e MO) em função da perda de água, entretanto, no método frito em óleo, o grande aumento no percentual de lipídeos é decorrência da incorporação do óleo de fritura ao filé durante o cozimento. Esse comportamento de resultados é semelhante aqueles descritos por: Badiani et al. (1998) em carne de cordeiros; Badiani et al. (2002) em cortes bovinos; García-Arias et al. (2003) em sardinha; Rosa (2003) em peito e coxa de frangos.
As médias de cinzas foram mais elevadas $(\mathrm{P}<0,05)$ nos filés assados em microondas e nos filés crus $(5,18$ e $5,01 \%$, respectivamente) do que nos filés assados em forno elétrico e frito em óleo (4,60 e 4,28\%, respectivamente) e, nesses tratamentos (FE e FO) as médias foram mais elevadas do que os filés cozidos em água. Analisando os resultados, verifica-se que o cozimento resulta em redução nos percentuais de cinzas ou perda de minerais para o meio, embora isso não tenha ocorrido nos filés preparados no forno de microondas. Dados com compotamento semelhante foram reportados por Rosa (2003) em peito de frango, entretanto para o corte coxa, esses resultados se comportaram de forma diferente. Gall et al. (1983), trabalhando com filés de pescado, relataram que na matéria seca, os filés cozidos em água e assados em microondas apresentaram médias de cinzas mais baixas do que a média dos filés crus.

Os tratamentos de cozimento resultaram $(\mathrm{P}<0,05)$ em aumento no teor de colesterol nos filés. As médias nos 
tratamentos CA e FE (46,89 e 46,46\%, respectivamente) foram mais elevadas do que as médias dos tratamentos FO e MO (54,78 e 57,28\%, respectivamente), mais elevadas do que o colesterol dos filés crus $(33,00 \%)$. Esses dados demonstram que houve aumento no teor de colesterol quando os filés foram cozidos, possivelmente resultado do aumento da matéria seca ou perda de água durante o cozimento Relatos semelhantes foram realiados por Badiani et al. (2002), Rao et al. (1996), Rhee et al. (1996) e Rosa (2003). Entretanto, alguns estudos relatam a diminuição do colesterol total após o cozimento. Mai et al. (1978) observaram redução no teor de colesterol em filés de peixes grelhados, assados e fritos, e Candela et al. (1997) verificaram redução entre 15 e $22 \%$ entre três espécies de peixes e associaram a perda de colesterol à sua eluição no óleo de fritura.

Com relação ao total de ácidos graxos saturados, o tratamento frito em óleo $(16,30 \%)$ reduziu $(\mathrm{P}<0,05)$ os percentuais de ácidos graxos saturados, quando comparado aos tratamentos CA, FE e MO $(31,92 ; 31,51$; $31,46 \%$, respectivamente) e ao controle $(32,55 \%)$. Essa redução no tratamento $\mathrm{FO}$ pode ser explicada pela possível incorporação do óleo de fritura aos filés durante o cozimento, pois o óleo de soja utilizado apresentou 14,04 $\%$ de ácidos graxos saturados (Tabela 1).

Considerando o total de ácidos graxos monoinsaturados foi observada uma redução $(\mathrm{P}<0,05)$ quando comparando aos filés fritos em óleo $(16,30 \%)$ com os filés CA, FE, MO e Crus (38,35; 40,87; 40,57 e 40,32\%, respectivamente). Possivelmente essa redução seja resultado da incorporação no filé de ácidos graxos monoinsaturados provenientes do óleo de fritura, cujo percentual foi de 20,16\% (Tabela 1).

$\mathrm{O}$ total de ácidos graxos poliinsaturados em filés do tratamento FO foi $(\mathrm{P}<0,05)$ superior $(57,00 \%)$ as médias encontradas nos filés dos tratamentos Cru, CA, FE e MO (22,97; 23,49; 22,30 e 23,53\%, respectivamente). Esse aumento no total de ácidos graxos poliinsaturados possivelmente seja decorrente da incorporação do óleo de soja, (que continha 67,37\% de ácidos graxos poliinsaturados) aos filés de tilápia.

O total de ácidos graxos da família ômega-3 e ômega-6 também mostraram aumento $(\mathrm{P}<0,05)$ nos filés fritos, contudo, o aumento na porcentagem de ômega $6(6,06 \%)$ foi maior que o aumento do ômega $3(50,94 \%)$. Esse aumento possivelmente seja decorrência da incorporação do óleo de soja aos filés fritos em óleo (Tabela 1). Dados semelhantes foram relatados por Agren \& Hänninem (1993) que, trabalhando com trutas submetidas à fritura em óleo, mencionam um aumento de $12 \%$ para o total de ácidos graxos da família ômega-3 e aumento de $27 \%$ para os ácidos graxos da família ômega-6.

$\mathrm{O}$ efeito biológico dos ácidos graxos essenciais depende da razão dos ácidos graxos das famílias ômega-6/ ômega-3, e em função disso, alguns autores consideram que a razão ômega-6/ômega-3 ideal é de 10-11:1 (SIMOPOULOS, 1991). Entretanto, a Japan Society for Lipid Nutrition recomenda que essa razão seja de 4:1 para adultos saudáveis e a The World Health Organization recomenda razões de ômega-6/ômega-3 entre 3:1 e 4:1 (ODA, 2002).

Analisando os dados de uma forma geral observase que o método de cozimento frito em óleo determina importantes alterações no perfil de ácidos graxos de filés de tilápia, quando comparado aos métodos de cozimento cozido em água, assado em forno elétrico ou em forno de microondas. E além disso, essa alteração do total dos tipos de ácidos graxos (saturados, monoinsaturados e poliinsaturados) está relacionado com o total dos tipos de ácidos graxos presentes no óleo de fritura. Assim, o perfil de ácidos graxos do óleo de fritura é importante quando considerado os aspectos de nutrição saudável e quando esse método de cocção for eleito na preparação de pratos.

\section{CONCLUSÃO}

O tratamento frito em óleo promove aumento na matéria seca e aumento no teor de lipídeos totais, resultado da maior perda de água e incorporação de óleo do meio de cozimento.

O método de cozimento frito em óleo altera as médias totais dos diferentes tipos de ácidos graxos decorrente do contato direto dos filés com o meio de cocção (óleo), cujas alterações estão relacionadas com os totais dos diferentes tipos de ácidos graxos presentes no óleo.

\section{REFERÊNCIA BIBLIOGRÁFICA}

AGREN, J. J.; HÄNNINEM, O. Effects of cooking on the fatty acids o three fresh water species. Food Chemistry, Great Britain, v. 46, n. 5, p. 377-382, 1993.

\section{ASSOCIATION OF OFFICIAL ANALYTICAL CHEMISTS. Official methodologies for analysis of the Association of Official Analytical Chemistrs. 15. ed. Arlington, 1990.}

BADIANI, A.; NANNI, N.; GATTA, P. P.; BITOSSI, F.; TOLOMELLI, B.; MANFREDINI, M. Nutrient content and retention in selected cuts from 3 month old ram lambs. Food Chemistry, Great Britain, v. 61, n. 1/2, p. 89-100, Jan./Feb. 1998. 
BADIANI, A.; STIPA, S.; BITOSSI, F.; GATTA, P. P.; VIGNOLA, G.; CHIZZOLINI, R. Lipid composition, retention and oxidation in fresh and completely trimmed beef muscles as affected by commom culinary practices. Meat Science, Oxford, v. 60, n. 2, p. 169-186, Feb. 2002.

BRAGAGNOLO, N.; RODRIGUES-AMAYA, D. B. Teores de colesterol em carne suína e bovina e efeito do cozimento. Ciência e Tecnologia de Alimentos, Campinas, v. 15, n. 1, p. 11-17, 1995 .

CANDELA, M.; ASTÍASARÁN, I.; BELLO, J. Effects of fryingand warmholding on fatty acids and colesterol of sole (Solea solea), codfish (Gadus morrhua) and hake (Merluccius merluccius). Food Chemistry, Great Britain, v. 58, n. 3, p. 227-231, 1997.

FERREIRA, D. F. Análises estatísticas por meio do Sisvar para o Windows versão 4.0. In: REUNIÃO ANUAL DA REGIÃO BRASILEIRA DA SOCIEDADE INTERNACIONAL DE BIOMETRIA, 45., 2000, São Carlos, SP. Anais... São Carlos: UFSCar, 2000. p. 255-258.

FIRESTONE, D. Official methods and recommended practices of the americam oil chemists society. 4. ed. Champaingn: AOCS, 1998. (Método Ce 1-62).

FOLCH, J.; LESS, M.; STANLEY, S. A simple method for the isolation and purification of total lipids from animal tissues. Journal of Biological Chemistry, Baltimore, v. 226, n. 1, p. 497-509, May 1957.

GALL, K. L.; OTWELL, W. S.; KOBUGUER, J. A.; APPLEDORF, $\mathrm{H}$. Effects of four cooking methods on the proximate, mineral and fatty acid composition of fish fillets. Journal of Food Science, Chicago, v. 48, n. 4, p. 1068-1074, July/Aug. 1983.

GARCÍA-ARIAS, M. T.; PONTES, E. A.; GARCÍALINARES, M. C. Cooking-freezing-reheating (CFR) of sardine (Sardina pilchardus) fillets: effect of different cooking and reheating procedures on the proximate and fatty acid compositions. Food Chemistry, Great Britain, v. 83, n. 3, p. 349-356, Nov. 2003.

GOKOGLU, N.; YERLIKAYA, P.; CENGIZ, E. Effects of cooking methods on the proximate composition and mineral contents of rainbow trout (Oncorhynchus mykiss). Food Chemistry, Great Britain, v. 84, n. 1, p. 19-22, Jan. 2004.
HUTTER, C. D. D.; LANG, P. Multiple sclerosis: sunlight, diet, immunology and etiology. Medical Hypotheses, [S.1.], v. 46, n. 1, p. 67-74, Feb. 1996.

MAI, J.; SHIMP, J.; WEIHRAUC, J.; KINSELLA, J. E. Lipids of fish fillets: changes following cooking by different methods. Journal of Food Science, Chicago, v. 43, n. 6, p. 1669-1674, Nov./Dec. 1978.

ODA, S. H. I. Diferentes métodos de abate e sexo na qualidade da carne de capivara (Hydrochaeris hydrochaeris L. 1966). 2002. 145 p. Dissertação (Mestrado em Zootecnia) - Universidade Federal de Lavras, Lavras, 2002.

POTTER, N. N.; HOTCHKISS, J. H. Ciência dos Alimentos. 5. ed. Zaragoza: Acribia, 1995. 667 p.

PUWASTIEN, P. et al. Proximate composition of raw and cooked thai freshwater and marine fish. Journal of Food Composition and Analysis, Thailand, v. 12, n. 1, p. 9-16, Mar. 1999.

RAO, V. K.; KOWALE, B. N.; BABU, N. P.; BISHT, G. S. Effect of cooking and storage on lipid oxidation and development of cholesterol oxidation products in water buffalo meat. Meat Science, Oxford, v. 43, n. 2, p. 179-185, June 1996.

RHEE, K. S.; ANDERSON, L. M.; SAMS, A. R. Lipid oxidation potential of beef, chicken, and pork. Journal of Food Science, Chicago, v. 61, n. 1, p. 8-12, 1996.

ROSA, F. C. Composição química e métodos de cocção de carcaça de frangos de corte alimentados com rações suplementadas com ômega-3. 2003. 131 p. Tese (Doutorado em Ciência dos Alimentos) - Universidade Federal de Lavras, Lavras, 2003.

SINCLAIR, H. M. The diet of Canadian Indians and Eskimos. Proceedings of the Nutrition Society, London, v. 12, n. 1, p. 69-82, 1953.

SIMOPOULOS, A. P. Summary of the nato advanced research workshop on dietary $\varpi-3$ and $\varpi-6$ fatty acids: biological effects and nutritional essentiality. American Institute of Nutrition, Philadelphia, v. 22, p. 521-526, 1991. 